\title{
GENERALIZATIONS OF SOME PROPERTIES OF CONVEX FUNCTIONS
}

\section{VLAD CIOBOTARIU-BOER}

Abstract. In this paper, generalizations of some properties of convex functions proved in $[4,5]$ are given.

Mathematics subject classification (2000): 26A51.

Keywords and phrases: Convex functions, Hlawka's inequality, Popoviciu's inequality.

\section{REFERENCES}

[1] D. Adamović, Généralisation d'une identité de Hlawka et l'inégalité correspondante, Mat. Vesnik, 1(16) (1964), 39-43

[2] J. V. Baston, On some Hlawka-type inequalities of Burkill, J. London Math. Soc., 12(2) (1976), 402-404.

[3] J. C. BURKILL, The concavity of discrepancies in inequalities of the means and of Hölder, J. London Math. Soc., 7(2) (1974), 617-626.

[4] V. Ciobotariu-Boer, On a property of convex functions, General Mathematics, Vol. 15, No. 1 (2007), 111-126.

[5] V. Ciobotariu-Boer, Some inequalities for convex functions, Octogon Mathematical Magazine, 15(1) (2007), 113-122.

[6] D. Z. Doković, Generalizations of Hlawka's inequality, Glasnik Mat.-Fiz. i Astr., 18(3) (1963), $169-175$.

[7] A. LuPAş, Asupra unei inegalităţi pentru funcţii convexe, Gazeta Matematică - Perfecţionare metodică şi metodologică în matematică şi informatică, 3(1-2) (1982), 49-52.

[8] T. Popoviciu, Sur quelques propriétés des fonctions d'une ou de deux variables réelles, Mathematica (Cluj), 8 (1934), 1-85.

[9] T. Popoviciu, Sur l'approximation des fonctions convexes d'ordre superieur, Mathematica (Cluj), 10 (1934), 49-54.

[10] T. Popoviciu, Sur certaines inégalités qui caractérisent les fonctions convexes, Analele Ştiinţifice Univ. “Al. I. Cuza” Iaşi, Secţia Mat., 11 (1965), 155-164.

[11] K. TODA, A method of approximation of convex functions, Tôhoku Math. J., 42 (1936), 311-317.

[12] P. M. VAsić AND Lu. R. STANKović, Some inequalities for convex functions, Math. Balcanica, 6(44) (1976), 281-288. 